\title{
Uma tecnologia social baseada em Crowdsourcing para busca de pessoas desaparecidas e cadastro de moradores de rua
}

\author{
Priscila Francielle Knoop Silveira ${ }^{1}$, Rodrigo Remor Oliveira ${ }^{1}$, Rodrigo Ruas \\ Oliveira $^{2}$ \\ ${ }^{1}$ IFSul - Campus Sapucaia do Sul, ${ }^{2}$ UFRGS \\ priscilaknoop@outlook.com, remor@sapucaia.ifsul.edu.br, \\ ruasoliveira@inf.ufrgs.br
}

\begin{abstract}
Resumo. Este artigo descreve Vox, uma plataforma que amplia a divulgação de desaparecidos, moradores de rua e abrigados, a fim de auxiliar na busca e na reinserção destas pessoas na sociedade. Vox é uma plataforma colaborativa que oferece um banco autossustentável para busca de pessoas. Vox permite a visualização de fotos, cadastro e criação de perfis com atributos físicos detalhados, inserção de descrições acerca das circunstancias de avistamento e desaparecimento, e mapeamento dos lugares em que a pessoa foi supostamente vista. A plataforma visa a tornar-se um banco de dados nacional e uma referência na busca por desaparecidos, moradores de rua e abrigados.
\end{abstract}

\begin{abstract}
This paper describes a platform--namely Vox-- that increases the dissemination of information regarding missing and homeless people, in an attempt to aid their reintegration into society. Vox is a collaborative platform that provides a self-sustainable database for searching people. Vox allows visualization of photos, registration, the creation profiles with detailed physical attributes, the description of the circumstances in which a person disappeared or was spotted, and the mapping of the places in which the person was allegedly spotted. The project aims at becoming a national database and a reference in the search for missing and homeless people.
\end{abstract}

\section{Introdução}

Cerca de 200 mil pessoas desaparecem anualmente no Brasil [Lima, 2011]. As causas são diversas: idosos acometidos por perdas de memória; adolescentes que fogem de casa ou de abrigos e outras instituições públicas; adultos que optam por deixar suas casas, empregos e rotinas; dependentes químicos; além de vítimas fatais de acidentes, calamidades ou crimes [Ferreira, 2013]. Além disso, em uma quantidade significativa dos casos, os desaparecidos não têm capacidade para voltar para casa por contra própria. Em uma pesquisa realizada por Garcia (2011), em 302 famílias, foi observado que aproximadamente $10 \%$ dos desaparecidos apresentavam algum tipo de deficiência física ou intelectual. Esse fato é alarmante pois dependendo da deficiência, o desaparecido pode não ser capaz de fornecer informações pessoais ou as de um contato próximo. Nessas situações e em muitas outras, tais como dependência química e idosos com perdas de memória, é bastante comum que os desaparecidos acabem à margem da sociedade, inseridos em populações em situação de rua ou encaminhados a abrigos, aumentando ainda mais a população em situação de vulnerabilidade social presente no país [Mattos 
and Ferreira, 2005]. Em um relatório feito pela Delegacia de Desaparecidos de Florianópolis em 2013, a guarda municipal constatou que em uma busca inicial dentre 250 moradores de rua, 40 deles foram identificados como pessoas desaparecidas que estavam sendo procuradas por suas famílias em Florianópolis e pelo restante do estado [Souza, 2013].

Para piorar a situação, a sociedade brasileira é extremamente ineficiente ao tentar lidar com o problema dos desaparecidos. Casos de desaparecimentos são investigados por delegacias, órgãos de administrações públicas e instituições não governamentais, como, por exemplo, a "SOS Desaparecidos". Entretanto, muitos casos acabam arquivados em delegacias, seja pela crença de que tais pessoas deixaram suas casas sem intenção de retornar, seja pela falta de interligação entre as diversas entidades. A título de comparação, os Estados Unidos possuem o sistema de alerta America's Missing: Broadcast Emergency Response, resultado de um trabalho conjunto entre sociedade civil, meios de comunicação e agências governamentais norte-americanas para os casos de desaparecimento infanto-juvenil. Tal projeto mostrou-se extremamente ágil para os casos de divulgação e busca de crianças desaparecidas [Garcia, 2011].

Portanto, diante da falta de interligação entre instituições e da quantidade imensa de pessoas que desaparecem todos os anos, fica evidente a falta de algo que possibilite a comunicação entre esses diferentes setores. Surge assim o seguinte questionamento: como seria possível correlacionar as informações das pessoas desaparecidas com as dos moradores de rua de uma maneira rápida e viável?

O presente artigo apresenta uma plataforma colaborativa a fim de facilitar a divulgação de desaparecidos, moradores de rua e abrigados, e também auxiliar na busca de desaparecidos e na reinserção de moradores de rua à sociedade. A plataforma, denominada Vox, possui os três seguintes objetivos específicos.

- Colaboração. Vox permite que os próprios usuários do sistema insiram informações sobre as pessoas, estejam elas desaparecidas, em situação de rua, ou abrigadas. Com isso, Vox pretende que a própria sociedade coopere com a procura e reinserção de pessoas à mesma. Além disso, Vox oferece dois possíveis tipos de cadastro: cadastro de pessoas (com descrição precisa ou aproximada da mesma) e cadastro de avistamentos (com indicação de local e descrição da circunstância).

- Busca rápida e facilitada. Vox possui um banco de dados com opções de busca de desaparecidos e correlação automática de informações acessível a qualquer pessoa com um navegador ou celular. Dessa forma, Vox pretende agilizar a reinserção das pessoas na sociedade e diminuir a invisibilidade social da população de moradores de rua

- Comunicação. Vox auxilia na comunicação entre diversas instituições, sejam elas governamentais ou não, e incentiva o uso de plataformas crowdsourcing sociais. Em outras palavras, Vox visa a incentivar o conceito de plataformas autoalimentadas pela comunidade e aplicadas à mesma na resolução de um problema social.

Primeiramente, o projeto pretende permitir que sejam cadastradas diversas pessoas, estejam elas desaparecidas, em situação de rua, ou abrigadas. Com isso, possibilitar que a própria sociedade coopere com a procura e reinserção de pessoas à sociedade, seja através de cadastro de pessoas, assim como cadastro de avistamentos. 
Pretende também tornar a busca de desaparecidos e a correlação de informações mais rápida e prática, diminuindo a invisibilidade social da população em situação de rua, e incentivar sua reinserção à sociedade. Auxiliar na comunicação entre diversas instituições, sejam elas governamentais ou não, e incentivar o uso de plataformas Crowdsourcing, ou seja, a utilização do conceito em que as informações da plataforma são fornecidas pela própria comunidade e incentivar o uso de tecnologias sociais, isto é, a utilização de plataformas aplicadas para a resolução de um problema social.

\section{Trabalhos relacionados}

Entre os sistemas relacionados encontrados destacam-se diversos sites que fazem a divulgação de desaparecidos, e alguns aplicativos com o mesmo fim. Porém, até o momento não há qualquer site que divulgue fotos de moradores de rua ou abrigados, e tampouco existem aplicativos voltados para essa população. Na Tabela 1 podemos ver uma comparação entre sistemas similares ao proposto pela plataforma, considerando aspectos tais como: se permite a divulgação de pessoas desaparecidas; se correlaciona as informações das pessoas desaparecidas com as dos moradores de rua; se o sistema é aberto para qualquer usuário fazer cadastros (colaborativo); se possui um banco de dados nacional para inserção de qualquer pessoa; e se permite que um usuário informe um avistamento de uma pessoa desaparecida.

Tabela 1. Tabela comparativa de sistemas relacionados

\begin{tabular}{|l|c|c|c|c|c|}
\hline & $\begin{array}{c}\text { Civulgação de } \\
\text { desaparecidos }\end{array}$ & $\begin{array}{c}\text { Correlação } \\
\text { das } \\
\text { informações }\end{array}$ & $\begin{array}{c}\text { Sistema } \\
\text { colaborativo }\end{array}$ & $\begin{array}{c}\text { Banco de } \\
\text { dados } \\
\text { nacional }\end{array}$ & $\begin{array}{c}\text { Cadastro de } \\
\text { avistamentos }\end{array}$ \\
\hline PLID & $\mathrm{V}$ & $\mathrm{X}$ & $\mathrm{X}$ & $\mathrm{X}$ & $\mathrm{X}$ \\
\hline ReDESAP & $\mathrm{V}$ & $\mathrm{X}$ & $\mathrm{V}$ & $\mathrm{X}$ & $\mathrm{X}$ \\
\hline Sinesp Cidadão & $\mathrm{V}$ & $\mathrm{X}$ & $\mathrm{X}$ & $\mathrm{X}$ & $\mathrm{X}$ \\
\hline Find People & $\mathrm{V}$ & $\mathrm{X}$ & $\mathrm{V}$ & $\mathrm{V}$ & $\mathrm{X}$ \\
\hline
\end{tabular}

O Programa de Localização e Identificação de Desaparecidos (PLID), desenvolvido pelo Núcleo de Apuração Criminal (NAC) do Ministério Público do Estado do Rio de Janeiro, trata de um banco de dados de desaparecidos, que procura auxiliar na busca e identificação destas pessoas. Ele cruza informações de diversos órgãos, como o Detran, o Disque Denúncia, o Departamento de Polícia Rodoviária Federal, e a Polícia Civil e Militar do Rio de Janeiro, entre outros [MP-RJ, 2015]. Distancia-se do projeto aqui abordado por ser voltado para o Rio de Janeiro e abordar apenas o tema de desaparecidos, não aderindo a instituições que acolhem moradores de rua.

O aplicativo Sinesp Cidadão, disponibilizado pela Secretaria Nacional de Segurança Pública do Ministério da Justiça, possui a funcionalidade de acesso a informações sobre pessoas desaparecidas. As informações utilizadas no aplicativo pertencem ao banco de dados de delegacias especializadas de alguns estados, sendo que três estados aderiram à aplicação até o momento [Ministério da Justiça, 2015]. Distanciase do projeto por não considerar a relação com moradores de rua, e também por ser dependente das delegacias de desaparecidos. Isso faz com que o usuário comum não possa cadastrar desaparecidos de forma independente, e torna o aplicativo obsoleto em estados que não aderiram ao projeto. 
A Rede Nacional de Identificação e Localização de Crianças e Adolescentes Desaparecidos (ReDESAP), é um site voltado também para a divulgação de desaparecimentos, focando em crianças e adolescentes. É um site-cadastro, que objetiva uma base única de dados de crianças e adolescentes desaparecidos [Secretaria de Direitos Humanos, 2015]. O site diferencia-se do projeto por abranger apenas crianças e adolescentes desaparecidos, ou seja, não considera adultos e a relação de desaparecidos com a população em situação de rua.

O Find People é um aplicativo produzido pela startup IlhaSoft, que visa auxiliar na busca de desaparecidos através de um banco nacional. Qualquer pessoa pode cadastrar um desaparecido, e o aplicativo traz um mapa com pessoas cadastradas perto do usuário [Campi, 2013]. O aplicativo permite ao usuário adicionar novos desaparecidos, mas também se distancia do projeto por não considerar a relação com moradores de rua. Apesar disso, a função de disponibilizar um mapa com pessoas cadastradas perto do usuário torna o aplicativo mais interativo que os demais.

Dentre os trabalhos apresentados, é possível verificar que nenhum possibilita a correlação de informações sobre desaparecidos com as de moradores de rua e abrigados. Também se verifica a necessidade de permitir que a população geral tenha um papel mais ativo na busca de pessoas. Isso seria viabilizado ao possibilitar que qualquer usuário cadastre avistamentos de pessoas que estão desaparecidas. Acredita-se que pelos motivos apresentados, nenhum dos trabalhos relacionados tornou-se um banco nacional para busca de pessoas. Diferentemente desses trabalhos, o Vox atende à todas as características analisadas.

\section{A plataforma Vox}

Esta seção descreve a plataforma Vox. Inicia-se com um resumo da pesquisa de campo efetuada para amparar o planejamento da plataforma. A seguir, apresenta-se uma visão geral da plataforma. Após, detalha-se cada um dos componentes da arquitetura.

\subsection{Pesquisa de Campo}

Para alcançar os objetivos da plataforma Vox, foram realizadas pesquisas de aprofundamento sobre este problema social para que a plataforma se adapte da melhor maneira possível ao o público alvo. Além da pesquisa dos trabalhos similares apresentados, foi realizada uma visita ao abrigo Bom Pastor, em Novo Hamburgo, RS, com o intuito de validar a proposta do projeto e encontrar possíveis pontos que necessitem de modificações.

A visita efetuada confirmou a necessidade de inserir informações físicas detalhadas das pessoas desaparecidas, para que ao adicionar um novo morador, seja possível verificar as pessoas com características similares, e possivelmente conseguir uma identificação. Além disso, também se se incluiu outros aspectos na busca (além do nome), tais como região.

\subsection{Visão Geral}

A plataforma usa o conceito de Crowdsourcing, que se encaixa no problema em questão, fazendo assim, a plataforma um projeto colaborativo. Sendo uma plataforma Crowdsourcing, o projeto propõe a cooperação e a comunicação direta entre familiares 
de desaparecidos, moradores de rua, ONG's e diversas instituições, sejam elas governamentais ou não.

Como pode ser visto na Figura 1, a arquitetura da plataforma Vox consiste em basicamente três componentes: o Vox mobile que representa um aplicativo desenvolvido para Android, o Vox web, que representa a aplicação Web, e o Vox server que representa o servidor que centraliza as informações oriundas do Vox mobile e Vox web.

A arquitetura foi planejada para suportar várias conexões ao mesmo tempo. A plataforma emprega Restfull Web Services através do framework Jersey, proporcionando rapidez no consumo e envio de dados entre os clientes e o Vox server. Além disso, o Restfull Web Services traz várias vantagens para a plataforma, tais como a reutilização de software, aumento da produtividade, interoperabilidade e escalabilidade. O banco de dados utilizado foi o MySQL, uma plataforma gratuita comumente utilizada no mercado. Para a persistência de dados no banco foi utilizado o framework Hibernate, juntamente com a especificação JPA (Java Persistence API), proporcionando assim maior facilidade ao trabalhar com bancos de dados relacionais e orientação a objetos. As próximas subseções descrevem em detalhes cada componente do modelo.

As requisições efetuadas pelos clientes são trafegadas utilizando o protocolo HTTP (Hypertext Transfer Protocol) e utilizando JSON (JavaScript Object Notation) para uma formatação leve de troca de dados, proporcionando rapidez à plataforma.

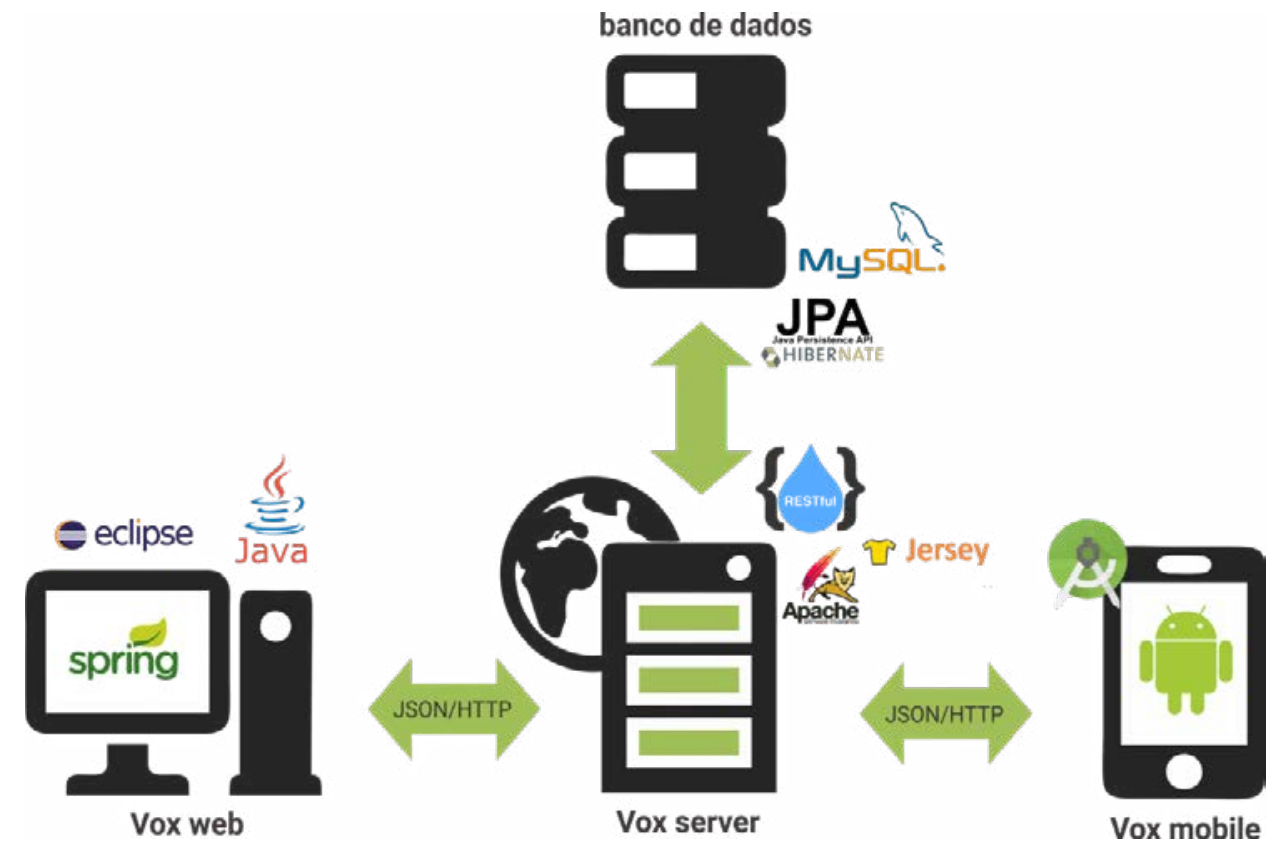

Figura 1. Arquitetura do sistema

\subsection{Vox server}

Para possibilitar a persistência de dados da aplicação foram utilizadas diversas ferramentas. Com a utilização dessas tecnologias foi possível que as classes demarcadas com a anotação @Entity fossem facilmente persistidas no banco, e que os relacionamentos entre classes também fossem facilmente definidos. É nessa camada que se define que cada objeto da classe Usuario possui um relacionamento com a classe PessoaProcurada, e que cada novo cadastro de pessoas deve informar qual usuário foi responsável pela ação. 
Com a finalidade de modelar as informações que serão armazenadas no banco de dados, foi criado um modelo lógico representado as tabelas necessárias ao sistema, seus atributos e seus relacionamentos. O modelo está ilustrado na Figura 2. No diagrama entidade relacionamento é possível observar que cada usuário (Usuario) pode possuir diversas informações de contato (InfoContato), e que um usuário pode cadastrar diversos desaparecidos, moradores de rua e abrigados (PessoaProcurada), assim como registros de avistamentos (Avistamentos) para uma determinada pessoa. Também é possível observar que um registro de avistamento obrigatoriamente deve possui circunstâncias detalhadas (Circunstancia) e uma localização (Localizacao).

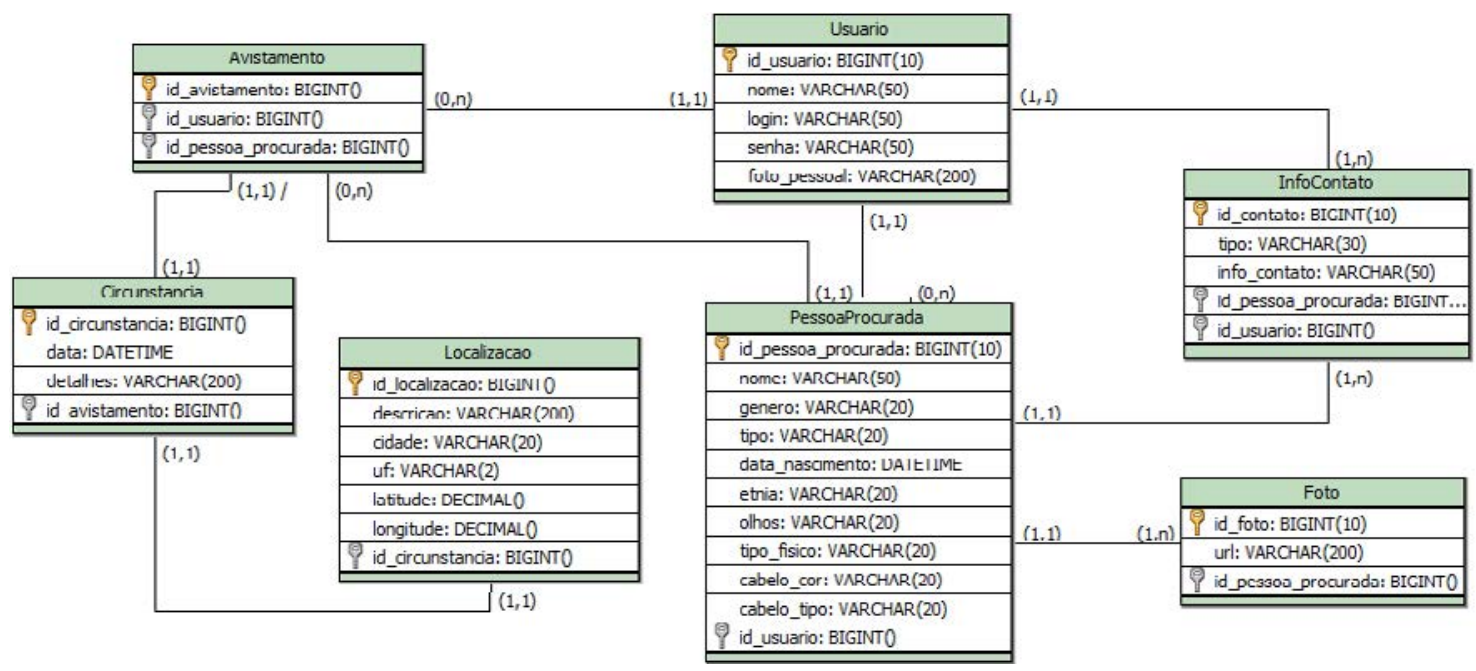

Figura 2. Modelo lógico do banco de dados

A camada de persistência está presente no Vox server componente que faz com que seja possível realizar a comunicação com o banco de dados. Esse componente está dividido em classes $D A O$ 's, Pojos e Services. A seguir será explicado mais sobre a camada de Services desse componente.

O padrão de persistência de dados DAO (Data Access Object) permite separar as regras de negócio das regras de acesso ao banco de dados. Todas as funcionalidades de um banco de dados, tais como inserir, buscar, atualizar e deletar objetos são manuseadas nessa parte da aplicação. Nela também estão todas as instruções, métodos, consultas e funções que possibilitam que a plataforma Vox insira novas pessoas e consiga acessá-las dependendo da demanda.

A plataforma realiza a comunicação entre os componentes através de um Web Services baseados em REST. Este por sua vez, utiliza o protocolo HTTP como protocolo de aplicação. Essa camada da aplicação proporciona uma comunicação rápida entre serviços e clientes, através de requisições que são transmitidas e recebidas através do formato JSON. Os recursos são dispostos em um servidor REST através de diversos links que levam o usuário à diversos estados da aplicação.

Para criar um Web Service Restful foi usada a especificação JAX-RS (Java API for RESTful Web Services), utilizada juntamente com a implementação Jersey. O JAXRS possui um foco em POJOS (Plain Old Java Object), oferecendo um conjunto de anotações que podem ser utilizadas. O HTTP é assumido como protocolo de aplicação, permitindo a utilização dos diferentes métodos de requisição utilizados no protocolo, tais como GET, POST, PUT e DELETE. A API também permite definir diversos tipos de 
conteúdo que os métodos produzem e consumem, tais como XML ou JSON. Para utilizar facilmente esses tipos de conteúdo, a aplicação também utiliza a JAXB (Java Architecture for XML Binding), que fornece uma API, as ferramentas e um framework para mapear objetos Java e documentos XML ou JSON.

No Vox server há diversos recursos que utilizam diferentes métodos de requisições e representam diferentes ações na aplicação. Por exemplo, no cadastro de novas pessoas procuradas do sistema Vox, é utilizado um método POST para que quando o Vox mobile acesse a URI "/pessoaProcurada" o servidor insira uma nova pessoa no banco de dados. Todas as requisições utilizam o formato de dados JSON, reconhecido por sua simplicidade e rapidez na troca de dados.

Sendo assim, além dessas ferramentas facilitarem o desenvolvimento da plataforma, também possibilitam uma facilidade de compreensão e manutenção do código. Um Web Service também independe de linguagens de programação, possibilitando uma interoperabilidade na aplicação e uma reusabilidade, pois seus recursos podem ser utilizados em diferentes aplicações.

\subsection{Vox mobile}

O componente Vox mobile é o componente apresentado ao usuário final, que permite acesso às funcionalidades da plataforma. Entre elas, o cadastro de contas, o cadastro de pessoas desaparecidas, moradores de rua e abrigados e também o cadastro de novos avistamentos dessas pessoas. Também a visualização de perfis, mapas com avistamentos recentes e a pesquisa por certas características.

Para que o Vox mobile faça o consumo do Vox server está sendo utilizada a classe RestTemplate do Spring framework, responsável pelo consumo do Vox server. O uso dessa classe simplifica o consumo do Vox server, sendo necessário informar apenas a URL do serviço requisitado, o tipo de retorno, o método de requisição do recurso e parâmetros quando existentes. Por exemplo, quando um usuário do Vox mobile entra no perfil de uma pessoa procurada, a aplicação requisita o método buscarPessoaPorId(). Para consumir esse método do Vox server, é necessário utilizar o método getForObject (URL, PessoaProcurada.class, id). Ao consumir esse método, também é necessário que o Vox mobile esteja preparado para receber as informações retornadas pelo Vox server. Para receber e enviar as informações no formato $J_{\text {son }}$ está sendo utilizada a biblioteca Google Gson em algumas partes do código para a serialização e deserialização de objetos. A biblioteca oferece métodos simples para a conversão de objetos (toJson () e fromJson ()), de objetos Java para representação Json, e vice e versa. A biblioteca permite, inclusive, a conversão de objetos complexos.

Para melhorar a experiência do usuário final, o Vox mobile utiliza o Material Design, um guia para design visual definido pela Google que pretende simplificar e unificar o layout dos aplicativos. O padrão inclui o uso de paletas de cores, ícones, animações, tipografias e hierarquias definidas pelo Material Design, objetivando que os aplicativos se tornem mais simples, claros e elegantes. O padrão também define recursos animados no uso de sombras e perspectiva, baseado na ideia de que um elemento flutue pela aplicação com sombra realista e profundidade próximas às propriedades de um material na vida real. O uso do padrão melhora a experiência do usuário, pois proporciona uma interface elegante e simples. Além disso, proporciona a sensação de familiaridade ao usuário pela proximidade do layout presente nas próprias ferramentas da Google. 
Além disso, a aplicação utiliza a API do Google Places para que o usuário possa adicionar novos avistamentos através de um campo autoComplete para endereços. Ao usuário digitar o endereço de um avistamento, ele recebe uma lista de possíveis endereços. Ao clicar em um item, o Vox mobile recebe a descrição do local, a cidade, estado e coordenadas, fazendo com que se consiga diversos dados com poucos cliques do usuário. O Vox mobile utiliza também a API do Google Maps para permitir a visualização de mapas nos perfis de pessoas procuradas e para a colocação de pontos que representam as últimas localizações dessas pessoas.

\subsection{Vox web}

A plataforma Vox possui uma aplicação Web para fins de divulgação do aplicativo Android e suas funcionalidades. Também possui informações sobre a plataforma e seus objetivos.

O framework utilizado neste componente é o Spring MVC, que segue o padrão MVC (Model View Controller), que é um modelo de arquitetura de software que separa a representação da informação e a interação do usuário. $O$ modelo possui três camadas: Model, View e Controller. A camada de Models consiste no encapsulamento dos dados da aplicação; A camada de Views é responsável por solicitar informações das Models necessárias para gerar representações de saída; A camada de Controllers é responsável por receber as requisições do usuário e executar determinadas ações.

Sempre que um usuário acessa a URL da página principal do Vox web, essa ação gera uma requisição para a HomeController, que recebe uma entrada e decide como processá-la. No caso dessa ação necessitar de acesso as regras de negócio da aplicação, a Controller requisita uma Model para tratar dessas regras. Ao fim disso, a HomeController requisita uma View para apresentar a saída do processo, enviando um retorno ao usuário na forma de um documento HTML referente a página inicial da aplicação.

As ideias principais do MVC são a reusabilidade de códigos e a separação dos diferentes conceitos utilizados, pois permite a separação da lógica de negócio, apresentação e navegação. A Figura 3 a seguir exemplifica uma requisição feita por um usuário, a comunicação da Controller com a Model para busca de informações, e a resposta retornada ao usuário através de uma View.

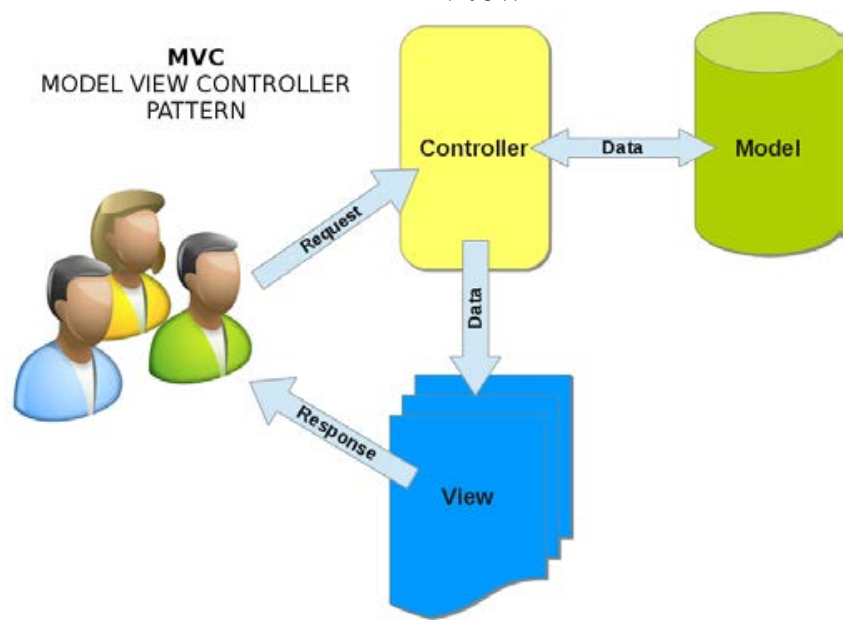

Figura 3. Estrutura de uma requisição utilizando MVC 


\section{Aspectos de avaliação}

$\mathrm{Na}$ etapa atual de avaliação do projeto, pretende-se apresentar o sistema em abrigos, albergues e instituições que possam estar interessadas na sua utilização. $O$ abrigo e albergue Bom Pastor, em NH, demonstrou interesse no projeto, e pretende-se apresentar o protótipo desenvolvido a outros locais que possam se beneficiar do uso da ferramenta.

Após a etapa de implementação do projeto, ocorrerá uma etapa de avaliação do protótipo. Pretende-se avaliar o protótipo através de questionários e entrevistas, considerando alguns critérios específicos. O protótipo desenvolvido será avaliado utilizando o Technology Acceptance Model, mais conhecido como modelo de aceitação de tecnologia (TAM). O TAM está fundamentado basicamente em dois construtos: a utilidade percebida e a facilidade de uso percebida, sendo que ambos medem completamente os efeitos das variáveis externas, como características do sistema, processo de desenvolvimento, treinamento, na intenção de uso (Silva, Pimentel, Soares; 2012). Sendo assim, os dados coletados em questionários e entrevistas serão avaliados utilizando esses dois critérios, ou seja, no quanto o usuário acredita que a ferramenta é útil e de fácil usabilidade.

\section{Discussão}

O Vox proporciona o cadastro de contas de usuários, que podem representar indivíduos ou uma organização. Para um usuário cadastrar uma conta é necessário que sejam informados alguns campos básicos, como e-mail, login e senha. Posteriormente um usuário pode alterar dados e cadastrar novas informações de contato. Usuário já cadastrados podem logar na plataforma informando o login e senha, e também podem fazer logout. Na Figura 4 está ilustrada a tela de login de usuários, que permite que o usuário escolha entre efetuar login na plataforma caso já seja cadastrado, ou cadastrar-se.
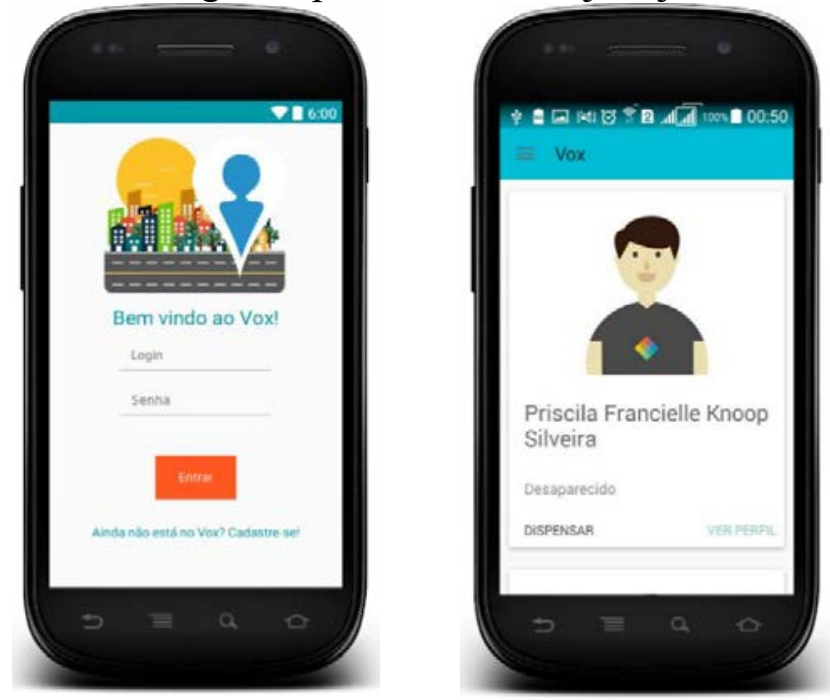

Figura 4. Tela de login e tela principal de divulgação de pessoas

A plataforma proporciona a divulgação de pessoas desaparecidas, moradores de rua e abrigados de acordo a localização do usuário logado. Além disso, na tela principal da aplicação são divulgadas pessoas a partir do critério de ordem de avistamento, ou seja, são exibidos no topo da lista pessoas cadastradas recentemente, ou que foram avistadas recentemente. Nessa tela o usuário possui a opção de "descartar" uma pessoa, isto é, 
passar o perfil e visualizar o seguinte, ou de visualizar suas informações na tela de perfil. Tela ilustrada na Figura 4.

A plataforma permite a visualização de perfis detalhados, com informações físicas e avistamentos mais recentes de pessoas desaparecidas, moradores de rua e abrigados. Permite também que qualquer usuário cadastre um desses perfis, informando características físicas detalhadas da pessoa a ser adicionada e o último avistamento dela. (Figura 5)
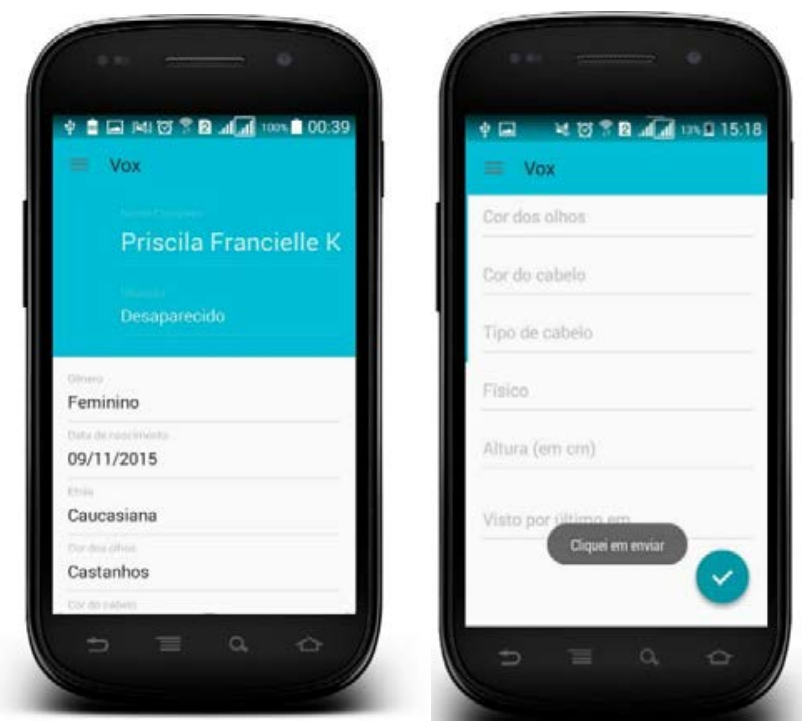

Figura 5. Tela de perfil e cadastro de pessoas

A plataforma permite que qualquer usuário cadastre um avistamento de uma pessoa desaparecida, morador de rua ou abrigado. Para isso, basta informar a data em que a pessoa foi supostamente vista, em que localidade ela estava e fornecer detalhes sobre as circunstâncias do avistamento. Cada usuário também possui um tipo de feed, em que é possível visualizar todas as pessoas que cadastrou, juntamente com a última vez em que elas foram vistas. Telas ilustradas na Figura 6.
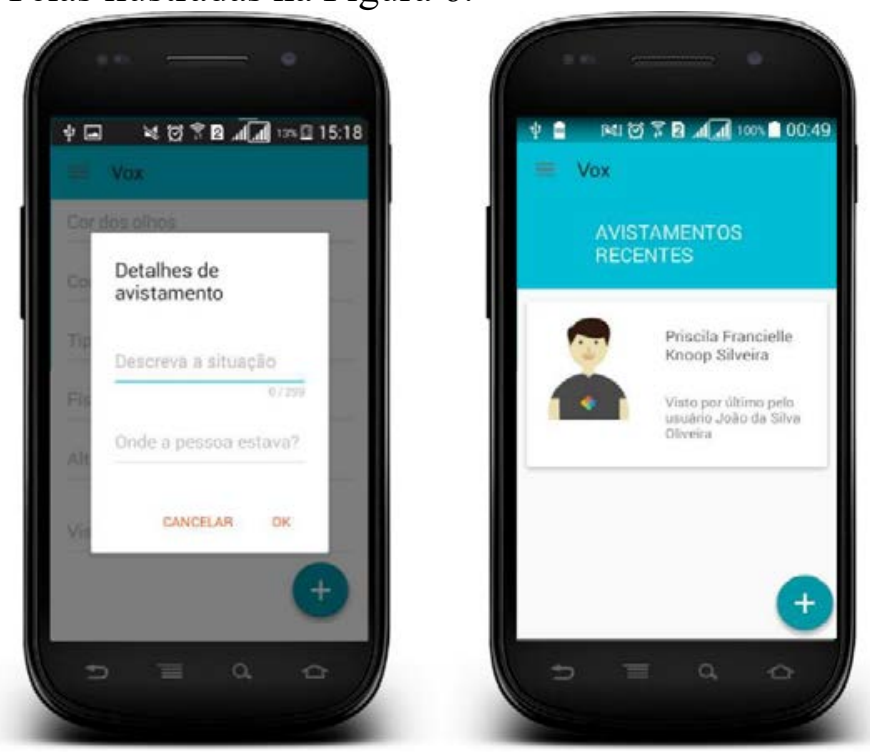

Figura 6. Telas de cadastro de avistamentos e avistamentos recentes 


\section{Conclusões e Trabalhos Futuros}

Com a presente pesquisa foi possível identificar que o tema do é pouco abordado e que não existem aplicações voltadas para o problema específico da relação entre pessoas desaparecidas e moradores de rua. Assim, este trabalho propôs uma plataforma para a diminuição de um problema relevante na sociedade por meio de uma tecnologia social.

Foram desenvolvidas funcionalidades visando facilitar a busca por desaparecidos e também diminuir a invisibilidade social da população de rua. A criação da plataforma colaborativa Vox possibilita uma interação de diversos setores, e também permite que a população civil tenha um papel mais ativo no processo de busca de pessoas. A fim de cumprir com os objetivos propostos, foram implementadas funcionalidades que possibilitam diminuir do impacto do problema apresentado através de um banco de dados nacional para busca de pessoas.

Dentre as tecnologias utilizadas, foi possível identificar que com a utilização do Web Service REST foi possível alcançar uma rapidez na troca de dados, essencial para aplicações móveis. Através do Framework Hibernate a persistência de dados foi desenvolvida de forma simples e prática. Quanto ao layout da plataforma, a utilização do padrão de layout Material Design proporcionou simplicidade e elegância à aplicação.

Atualmente, o projeto encontra-se em fase de avaliação e posteriormente irá para fase de divulgação e implementação em instituições de todos os estados brasileiros, a fim de acelerar o processo de criação de um banco nacional para busca de pessoas.

Entende-se que há dificuldades enfrentadas quando o proposto é uma plataforma colaborativa. Primeiramente, por possibilitar a inserção de dados pela população, há o problema de confiabilidade nos dados inseridos, que podem muito bem ser inseridos com a intenção de brincadeira ou de dificultar a utilização da aplicação. Além disso, sistemas colaborativos e tecnologias sociais não são imensamente utilizados, pois o fato de ajudar a aplicação e a sociedade não são motivações o suficiente para que um usuário utilize plataformas desse tipo.

Como trabalho futuro pretende-se adicionar funcionalidades que divulguem a plataforma, a tornem mais confiável e que também a tornem mais atrativa para o público em geral. Para isso, seria interessante desenvolver um sistema de avaliações de usuários, para que seja possível avaliar um usuário ou uma informação como confiável ou não, alertando para possíveis dados falsos; a inserção de selos, ou seja, pequenas premiações dentro da aplicação para usuários que sejam ativos na aplicação e de alguma corroboram para o banco de dados de busca, como forma de motivação; e também realizar uma campanha de divulgação do projeto para ONG's e instituições que trabalhem com moradores de rua, abrigados e também desaparecidos ao redor do país, para evitar que a aplicação se torne obsoleta em outros locais. Outro trabalho futuro seria integrar a aplicação com redes sociais, para que assim seja possível que cada perfil possua a opção de compartilhamento (compartilhar pessoas desaparecidas e avistamentos, por exemplo). Além de atrair mais usuários, isso facilitaria a divulgação de pessoas desaparecidas, moradores de rua e abrigados. Consequentemente, a adição de mais usuários aumentaria a quantidade de casos de desaparecimentos resolvidos, pois a divulgação não estará limitada à usuários da plataforma. 


\section{Referências}

Campi, M. (2013) “App brasileiro ajuda a encontrar pessoas desaparecidas", http://exame.abril.com.br/tecnologia/noticias/app-brasileiro-ajuda-a-encontrarpessoas-desaparecidas?page $=1$, agosto. 2015 .

Ferreira, L. (2013) "De problema de família a problema social: notas etnográficas sobre o desaparecimento de pessoas no Brasil contemporâneo", http://aa.revues.org/426, julho. 2015.

Garcia, C. (2011) "O que é o desaparecimento de crianças e adolescentes? ", http://www.moreirajr.com.br/revistas.asp?fase=r003\&id_materia=4546, junho. 2015.

Lima, V. (2011) "Desaparecidos: o descaso do Estado", http://www2.camara.leg.br/camaranoticias/radio/materias/REPORTAGEMESPECIAL/403230-DESAPARECIDOS--O-DESCASO-DO-ESTADO(05'46\%22).html, junho. 2015.

Mattos, R. e Ferreira, R. (2005) "O idoso em situação de rua: Sísifo revisitado", $<$ http://www.scielo.br/scielo.php?script=sci_arttext\&pid=S0103166X2005000100004\&lng=en\&nrm=iso $>$, julho. 2015.

Ministério da Justiça. (2015) “Aplicativo Sinesp Cidadão ganha módulo para localizar pessoas desaparecidas", http://www.justica.gov.br/noticias/aplicativo-sinesp-cidadaoganha-modulo-para-localizar-pessoas-desaparecidas, julho. 2015.

Ministério Público do Estado do Rio de Janeiro. (2015) "Programa de Localização e Identificação de Desaparecidos", http://plid.mprj.mp.br/apresentacao, julho. 2015.

Secretaria de Direitos Humanos. (2015) "Rede Nacional de Identificação e Localização de Crianças e Adolescentes Desaparecidos", http://www.desaparecidos.gov.br/index.php/redesap, julho. 2015.

Silva, P. e Pimentel, V. e Soares, J. (2012) "A utilização do computador na educação: aplicando $\quad 0 \quad$ Technology Acceptance Model (TAM).”, http://www.ies.ufpb.br/ojs/index.php/biblio/article/viewFile/14208/8113, julho. 2015.

Souza, C. (2013) "Moradores de rua estão na lista da delegacia de desaparecidos de Florianópolis", http://ndonline.com.br/florianopolis/noticias/128969-moradores-derua-estao-na-lista-da-delegacia-de-desaparecidos-de-florianopolis.html, junho. 2015. 